\title{
Androgen receptor CAG repeat length in Jewish Israeli women who are BRCA1/2 mutation carriers: association with breast/ovarian cancer phenotype
}

\author{
Efrat Dagan ${ }^{1,2}$, Eitan Friedman ${ }^{* 3,4}$, Tamar Paperna ${ }^{1}$, Nirit Carmi $^{3}$ and \\ Ruth Gershoni-Baruch ${ }^{1,5}$
}

${ }^{1}$ Institute of Human Genetics, Rambam Medical Center, Haifa, Israel; ${ }^{2}$ Department of Nursing, Faculty of Welfare and Health Sciences, University of Haifa, Israel; ${ }^{3}$ The Susanne Levy Gertner Oncogenetic unit, Danek Gertner Institute of Genetics, Sheba Medical Center, Tel-Hashomer, Israel; ${ }^{4}$ Sackler School of Medicine, Tel-Aviv University, Tel-Aviv, Israel; ${ }^{5}$ Technion-Institute of Technology, Rappaport Medical School, Haifa, Israel

$B R C A 1 / 2$ mutation carriers are at an increased risk for developing breast and/or ovarian cancer. Yet, the genetic and environmental factors that govern the phenotypic expression of mutant $B R C A 1 / 2$ alleles remain elusive. The CAG repeat within exon 1 of the androgen receptor $(A R)$ gene is reportedly associated with breast cancer phenotype in BRCA1 mutation carriers. Two hundred and twenty seven $B R C A 1 / 2$ mutation carriers were genotyped for the polymorphic $A R$ CAG repeat, and allele size was correlated with breast/ovarian cancer morbidity parameters. Of 227 BRCA1/2 carriers, 169 were $B R C A 1$ mutation carriers and 58 carried a BRCA2 mutation, 149 had breast and/or ovarian cancer and 78 were asymptomatic mutation carriers. The mean age at diagnosis in women with either or both neoplasms was $46.7 \pm 11.2$ years, and that of the asymptomatic group $-45.8 \pm 9.4$ years, a statistically insignificant difference. The $A R$ CAG repeat ranged from eight to 28 in all tested women, and the mean number of the repeats were not statistically different between affected $(18.3 \pm 2.4)$ and asymptomatic mutation carriers (18.6 \pm 2.1 ). The $A R$ CAG repeat among patients with early onset ( $<42$ years) breast cancer was significantly shorter $(17.5 \pm 2.3)$ compared with asymptomatic individuals $(18.6 \pm 2.1)(P<0.01)$, and the shorter allele - the younger the age at diagnosis. There is no conclusive evidence of association between $A R$ CAG repeat size and breast or ovarian cancer risk in Jewish BRCA1/2 mutation carriers. A small effect of a short $A R$ CAG allele size on breast cancer at early age ( $<\mathbf{4 2}$ years) cannot be excluded.

European Journal of Human Genetics (2002) 10, 724 - 728. doi:10.1038/sj.ejhg.5200880

Keywords: BRCA mutations; penetrance; modifier genes; androgen receptor; breast cancer

Introduction

BRCA1/2 mutation carriers have a lifetime risk of $40-80 \%$ for developing breast cancer and 16-36\% for developing ovarian cancer. $^{1-5}$ Three predominant mutations (185delAG and 5382insC in BRCA1 and 6174delT in $B R C A 2)$ are frequent among Jews of Ashkenazi descent with carrier frequencies of $2.5 \%$ in the general Ashkenazi popula-

${ }^{*}$ Correspondence: E Friedman; Chief, Susanne Levy Gertner Oncogenetics Unit, The Danek Gertner Institute of Genetics, Sheba Medical Center, TelHashomer, 52621, Israel. Tel: 972-3-530-3173, Fax: 972-3-535-7308; E-mail: eitan211@sheba.health.gov.il

Received 16 April 2002; revised 9 July 2002; accepted 24 July 2002 tion, ${ }^{6-8}$ about $12 \%$ in unselected Ashkenazi patients with breast cancer ${ }^{9}$ and $29 \%$ of unselected ovarian cancer patients of the same ethnic origin. ${ }^{10}$ While finding a germline mutation in BRCA1/2 genes is an accepted objective means of evaluating cancer risk, penetrance is incomplete. The factors that govern the phenotypic expression of mutant BRCA1/2 alleles, whether genetic or environmental, have not been fully elucidated. Several genes may parenthetically act as $B R C A$ modifiers with androgen receptor $(A R)$ being one candidate gene. The involvement of BRCA1 protein in steroid hormone regulation ${ }^{11}$ combined with the putative role that the polymorphic CAG repeat (encod- 
ing for a polyglutamine tract) in exon 1 of the $A R$ gene in prostate cancer ${ }^{12-14}$ contributed to the notion that $A R$ gene may be involved in breast cancer pathogenesis. Direct analyses of the CAG repeat number in women diagnosed with breast cancer under $40^{15}$ or 55 years of age, ${ }^{16}$ did not demonstrate an association between age at disease diagnosis and CAG repeat size. Recently, a protective effect of having a short $A R$ CAG repeat size allele was shown for Canadian incident breast cancer cases. ${ }^{17}$ Yet, in a study analysing 304 BRCA1 mutation carriers, Rebbeck and coworkers $^{18}$ reported that having an allele size longer than 28 repeats, is significantly associated with breast cancer phenotype. In order to evaluate the possible contribution of this polymorphism to mutant BRCA phenotype, we genotyped Jewish Israeli mutation carriers for the $A R$ CAG repeat size and correlated repeat size with relevant phenotypic features.

\section{Materials and methods}

Subjects and clinical data

Participants included 227 Jewish women, all carriers of one of the predominant mutations in $B R C A 1 / 2$. Study participants were ascertained and recruited via the Oncogenetic clinics at two medical centers, Rambam (Haifa) and Sheba (Tel-Hashomer), from October 1996 to June 2001. All study participants were considered to represent 'high-risk' families, and not incident cases. Women who were not high risk were not genotyped for BRCA1 BRCA2 mutations in the context of these oncogenetics clinics. All carriers underwent pretest genetic counselling and were subsequently genotyped for the $A R$ CAG repeats in an unselected manner. Data collected on each participant included demographics, type of cancer (based on pathological reports), age at diagnosis (for patients), last follow-up (for asymptomatic individuals) or age of prophylactic surgery (for asymptomatic individuals or patients) if any was performed, and family history of cancer. The study was approved by the institutional review boards at both centres, and each participant signed a written informed consent form.

\section{Genetic analyses}

DNA extraction Genomic DNA was extracted from peripheral blood leukocytes using standard techniques and employing a commercial kit (the Gentra system kit, Gentra Inc. Minneapolis MN, USA) according to the manufacturer's recommended protocol.

BRCA1/2 mutation analysis The three founder mutations: 185delAG, 5382insC and 6174delT (BRCA1/2) and the Tyr978X (BRCA1) mutation were detected by PCR amplification. Specific primers, that generate a modified restriction enzyme site, made to distinguish the wild type allele from the mutant allele by restriction enzyme digest, as previously described. ${ }^{2,19,20}$
AR CAG repeat length polymorphism analysis Fluorescent PCR primers, flanking the CAG repeats section within exon 1 of the $A R$ gene, were employed to PCRamplify the relevant genomic region, as previously described. ${ }^{13}$ Subsequent analysis was performed using the ABI prism 310 DNA sequencer (PE Biosystems, Foster City, CA, USA) and the Gene Fragment analyzer application for allele size determination.

\section{Statistical methods}

The study group was subdivided by phenotype to several subsets: asymptomatic women (AS), breast cancer (BC), ovarian cancer (OC), breast and ovarian cancer (BCOC) and early onset breast cancer patients (EOBC) (diagnosed at or under the age of 42 years). $A R$ genotype analysis involved dichotomising the total sample by using cut off points along the $A R$ CAG repeat length distribution. Specifically, the cut point for the short $(<18 / 18)$ and the long $(>19 / 19)$ alleles were performed by using the median number of CAG repeats in each allele. Short, Long and combined $A R$ CAG repeat number was compared between these phenotypically defined subgroups by using $t$-test or a one way ANOVA. Pearson correlation test was performed to find the possible association between the sizes of the $A R$ CAG repeats size and age at diagnosis among affected women. Analyses of disease free-survival were done using Cox proportional regression. Participants were followed up (retrospectively) from 20 years of age until one of several events occurred: invasive breast cancer, ovarian cancer, breast and ovarian cancer (for the age of diagnosis for the first malignancy of the two) and the age of interview for asymptomatic carriers. In all survival analyses, asymptomatic carriers were censored at the age of last follow up exam or at the age of prophylactic surgery (either mastectomy or oophorectomy) if ever performed.

AR CAG repeat genotype analyses Initially, we analysed the correlation of $A R$ CAG repeat number with phenotypic features on the group of affected patients as a whole, then separately on patients with breast cancer, early onset breast cancer and ovarian cancer. Subsequently, we analysed correlation of the $A R$ CAG genotype on the phenotype of 185delAG BRCA1 and 6174delT BRCA2 mutation carriers. This latter analysis was done since the 185 delAG mutation results in a truncated protein at codon 39; the $A R$ gene interaction site is abolished. ${ }^{21}$ This analysis could not be performed for the 5382insC mutation carriers separately, because of the small sample size $(n=37)$.

\section{Results}

\section{Patient's characteristics}

Two hundred and twenty seven women participated in this study, all either BRCA1 or BRCA2 mutation carriers. Of these, 169 were BRCA1 mutation carriers (185delAG, $n=130$; Tyr978X, $n=2$ and 5382insC, $n=37$ ) and 58 carried the 6174delT BRCA2 mutation. Unilateral breast cancer 
was diagnosed in 79 women, bilateral breast cancer (BBC) in 15 , ovarian cancer in 41 , breast and ovarian cancer in 14 and 78 were asymptomatic mutation carriers. Among women with breast cancer, 46 diagnosed before 42 years of age, and in three - ovarian cancer developed later. The mean age at diagnosis among affected women (either with breast, ovarian cancer or both neoplasms) was $46.7 \pm 11.2$ years and that of the asymptomatic group $-45.8 \pm 9.4$, a statistically insignificant difference.

\section{Genotype-phenotype correlation}

Table 1 summarises the results of $A R$ CAG repeats genotyping in all carriers by phenotypes. $A R$ CAG repeat number in our population ranged from eight to 28. Using one way ANOVA, the mean number of CAG repeats (short, long or combined alleles) was not statistically different between the various phenotypically subgroups: AS, BC, OC, EOBC and BCOC (Table 1). Using $t$-test, significant differences in mean numbers of repeats were demonstrated between AS carriers and women with EOBC for the short allele $(17.3 \pm 2.2$ compared with; $16.5 \pm 3.0 ; P=0.033)$, long allele $(19.8 \pm 2.7$ compared with; $18.8 \pm 2.4 P=0.044)$ and combined alleles (18.6 \pm 2.1 compared with; $17.5 \pm 2.3$; $P=0.01)$, respectively. The means of the $A R$ CAG repeats for the short, long and combined alleles, were significantly smaller in individuals with EOBC compared with asymptomatic carriers (Table 1).

$A R$ CAG repeat length was correlated with age at diagnosis of breast cancer: the shorter the $A R$ CAG repeat number
- the younger age at diagnosis (Table 2). For all affected individuals, the long and combined (but not the short) $A R$ CAG alleles, showed a significant correlation with age at diagnosis $(P=0.001 ; P=0.003$, respectively). For $\mathrm{BC}$ patients only, $A R$ alleles (short, long and combined) were significantly correlated with age at diagnosis. For OC patients, the long $A R$ alleles were significantly correlated with age at diagnosis. Other correlation between age at diagnosis and BBC and BCOC were not significant.

We estimated the effect of $A R$ CAG repeat number on diagnosis of any $B R C A$-related malignancy (breast and/or ovarian cancer). $A R$ CAG repeat number did not affect cancer risk (Hazard ratio $(\mathrm{HR})=1.2,95 \%$ CI $0.8-1.6$, for short allele and $\mathrm{HR}=1.4,95 \% \mathrm{CI} 1.04-2.0$, for long allele, not significant (ns)) (Figure $1 \mathrm{a}$ and $\mathrm{b}$ respectively). No effect of $A R$ CAG repeat number could be demonstrated when separate analyses were carried out for the phenotypically diverse subgroups, including asymptomatic mutation carriers. However, the short $A R$ allele was found to increase EOBC risk in 185delAG BRCA1 mutation carriers $(\mathrm{HR}=2.45$, 95\% CI 1.1-5.5) (Figure 2).

\section{Discussion}

This study does not provide conclusive evidence for an effect of the length of the polyglutamine tract in the transactivation domain of the $A R$ gene on the phenotypic expression of breast and/or ovarian cancer in Jewish BRCA1/2 mutation carriers. Notably, the mean and combined CAG $A R$ allele sizes were similar in asymptomatic

Table 1 AR CAG repeat length in BRCA1/2 mutation carriers

\begin{tabular}{|c|c|c|c|c|}
\hline \multirow[b]{2}{*}{ Disease status } & \multirow[b]{2}{*}{$\mathrm{n}$} & \multicolumn{3}{|c|}{$\mathrm{AR} C A G$ repeats (mean $\pm S D$; range) } \\
\hline & & Short allele & Long allele & Combined allele \\
\hline Asymptomatic & 78 & $17.3 \pm 2.2 ; 11-22^{a}$ & $19.8 \pm 2.7 ; 14-28^{a}$ & $18.6 \pm 2.1 ; 14-25^{b}$ \\
\hline Total affected & 149 & $17.0 \pm 2.3 ; 8-22$ & $19.7 \pm 2.9 ; 11-28$ & $18.3 \pm 2.4 ; 10-24$ \\
\hline Unilateral breast cancer & 79 & $17.2 \pm 2.2 ; 11-22$ & $19.7 \pm 2.5 ; 11-26$ & $18.4 \pm 2.3 ; 10-23$ \\
\hline Bilateral breast cancer & 15 & $15.9 \pm 3.0 ; 8-20$ & $19.6 \pm 2.5 ; 16-25$ & $17.8 \pm 2.3 ; 12-21$ \\
\hline Breast and ovarian cancer & 14 & $17.3 \pm 2.1 ; 13-21$ & $18.7 \pm 3.3 ; 14-28$ & $18.0 \pm 2.6 ; 13-24$ \\
\hline Of the above patients, early onset breast cancer ( $<42$ years) & 46 & $16.5 \pm 3.0 ; 11-21^{a}$ & $18.8 \pm 2.4 ; 11-26^{a}$ & $17.5 \pm 2.3 ; 10-23^{b}$ \\
\hline Ovarian cancer & 41 & $17.0 \pm 2.3 ; 11-22$ & $20.0 \pm 3.7 ; 11-28$ & $18.5 \pm 2.6 ; 11-24$ \\
\hline
\end{tabular}

${ }^{a} t$-test analysis between asymptomatic carriers and EOBC $(P<0.05) .{ }^{b}$-test analysis between asymptomatic carriers and EOBC $(P<0.01)$.

Table 2 Pearson correlation between $A R$ CAG number of repeats and age at diagnosis

\begin{tabular}{|c|c|c|c|c|c|c|c|c|}
\hline \multirow{2}{*}{ Disease status } & \multicolumn{2}{|c|}{ Age at diagnosis } & \multirow{2}{*}{\multicolumn{2}{|c|}{ Short }} & \multicolumn{2}{|c|}{ Alleles $^{\mathrm{a}}$} & \multirow{2}{*}{$\begin{array}{c}\text { Combined } \\
r\end{array}$} & \multirow{2}{*}{$P$} \\
\hline & & & & & & & & \\
\hline Total affected & 149 & $46.7 \pm 11.2$ & 0.10 & 0.145 & 0.28 & 0.001 & 0.24 & 0.003 \\
\hline Early onset breast cancer ( $<42$ years) & 46 & $36.3 \pm 4.8$ & 0.05 & 0.74 & 0.19 & 0.20 & 0.10 & 0.51 \\
\hline Ovarian cancer & 41 & $54.22 \pm 12.45$ & -0.094 & 0.56 & 0.41 & 0.008 & 0.25 & 0.12 \\
\hline
\end{tabular}

$\mathrm{r}=$ Pearson correlation analysis; $P=$ significance level. ${ }^{\mathrm{a}}$ Allele sizes were chosen in relation to the median. There is no known biological difference between the 18 or 19 repeat alleles. Comparison of the most extreme groups (longest vs shortest) yielded small numbers in each group for any statistical analysis to be carried out. 

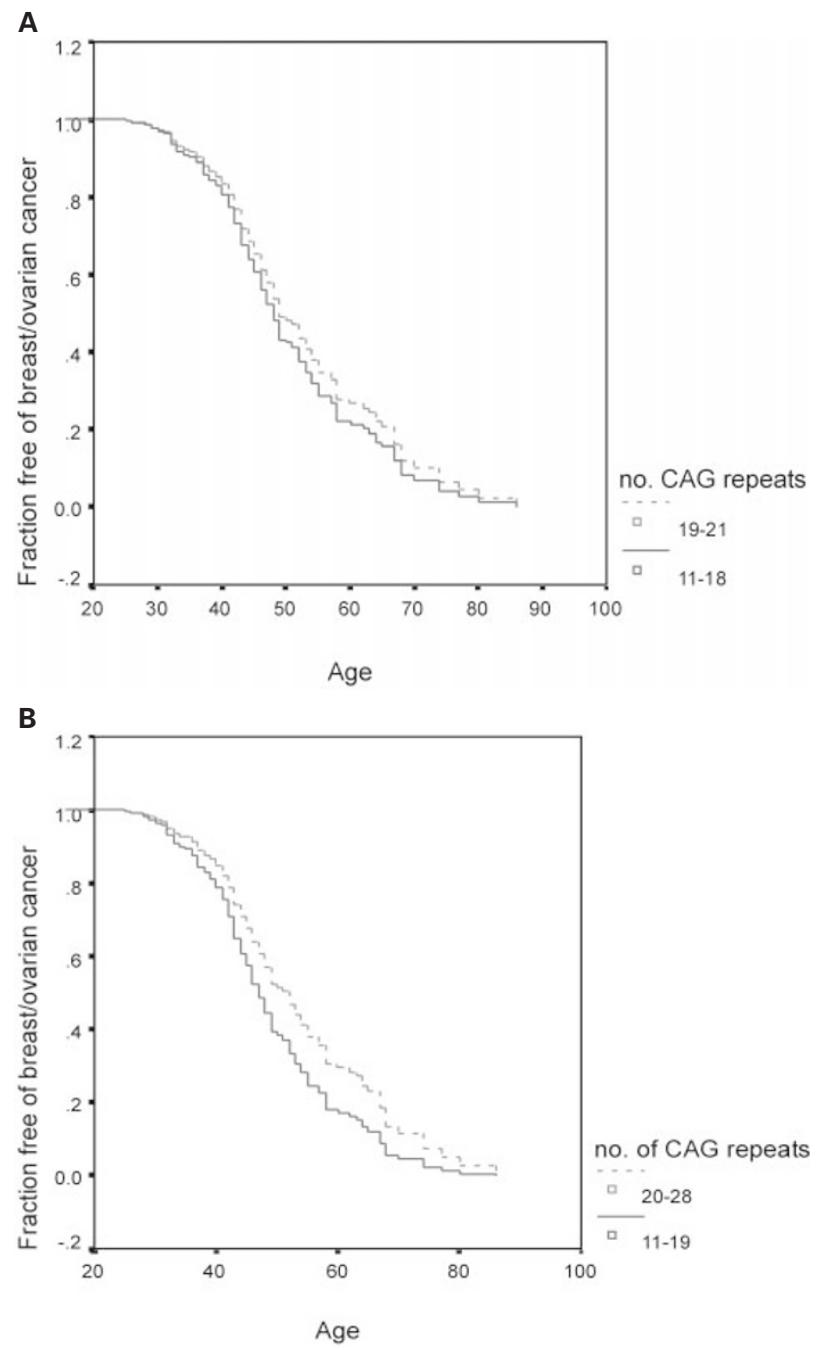

Figure 1 (a) Hazards Ratio (HR) associated with short AR CAG allele in $B R C A 1 / 2$ mutation carriers. (b) HR associated with long $A R$ CAG allele in $B R C A 1 / 2$ mutation carriers.

mutation carriers and in women who developed both breast and ovarian cancer. Rebbeck and coworkers ${ }^{18}$ reported that having more than 29 CAG repeats associated with earlier age of onset of breast cancer in BRCA1 mutation carriers. In our study, not a single individual had an allele longer than 28 CAG repeats, and there was no evidence for an increased risk of breast cancer for alleles with 29 or more repeats. Similarly, in another study from Israel analysing Jewish women, ${ }^{22}$ no effect of the CAG or GGC repeats within $A R$ gene could be shown in 188 BRCA1/2 mutation carriers ( 122 breast cancer cases and 66 asymptomatic individuals). These 188 individuals were all Jewish women recruited from among high risk families, similar to our study population. The differences in the effect of AR (CAG)n on BRCA1 phenotype between Jewish and non Jewish women need to be reconciled. These differences

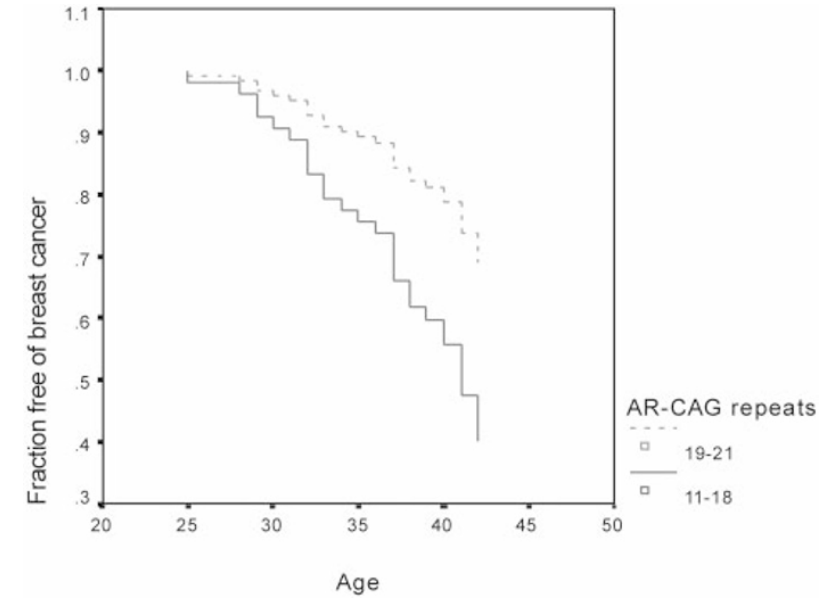

Figure 2 HR associated with short AR CAG allele in 72 185deIAG carriers; Early onset breast cancer $(n=32)$, asymptomatic (censored) individuals $(n=40)$.

may be related to the site of the specific pathogenic mutation within the BRCA1 gene, the time of follow-up, types of statistical analyses, and ascertainment bias.

A moderate effect of having a short $A R$ CAG allele size on breast cancer diagnosed at an early age (before 42 years) is suggested by our data. This trend was observed consistently by using different statistical analyses. The mean size of the $A R$ CAG repeat was significantly shorter among patients with early onset breast cancer compared with asymptomatic mutation carriers. Furthermore, among affected individuals with breast cancer, the smaller the number of CAG repeats - the earlier is the age at diagnosis. Similarly, a trend for an earlier age at diagnosis occurring with shorter AR CAG repeats was reported in study that analysed incident Jewish Ashkenazi ovarian cancer patients, in both sporadic cases and BRCA mutation carriers. ${ }^{23}$ Combined, these data suggest that AR plays a role in the pathogenesis and progression of breast and ovarian cancer in Jewish women, regardless of BRCA mutation status.

Biological significance of the CAG repeat length variation is suggested by in vitro studies, demonstrating that the shorter $A R$ CAG repeat exhibits greater transactivation capability. ${ }^{24}$ Given the endocrine dependency of breast cancer and the effect that androgenic pathway has on breast tissue proliferation, ${ }^{22,25}$ it is plausible that an increased androgen transactivation may promote uncontrolled breast epithelial tissue proliferation. As a further support for the putative role that the short CAG repeat plays in determining breast cancer phenotype, $\mathrm{Yu}$ et $a l^{26}$ suggested an association between shorter CAG repeat length and tumour grade and survival. Several studies reported of an association between the shorter $A R$ (CAG)n repeat and the risk for prostate cancer. ${ }^{25}$ The association between a short $A R$ CAG repeats size and age at diagnosis of breast cancer in the present study were most notable in 
185delAG BRCA1 mutation carriers. The resulting mutant $B R C A$ protein is a peptide of 39 amino acids, lacking the domain that presumably binds the $A R .^{21}$ While it is plausible that this is the mechanism by which $A R$ CAG repeat affects breast cancer phenotype in 185delAG mutation carriers, it remains speculative. One facile way to gain insight that would be to compare the effect of $A R$ CAG genotype on a large number of 5382insC mutation carriers, a mutation that leaves the $A R$-BRCA1 interaction domain intact.

Our data differ markedly from those reported for women with sporadic breast cancer, where a short $A R$ CAG allele seemed to confer protection from breast cancer. ${ }^{17}$ The differences in these observations may relate to the presence of mutant BRCA1 protein in our patients compared with sporadic cases.

Our study did not detect a single individual with $B R C A 1$ or BRCA2 mutation with more than 28 CAG repeats in the $A R$ gene. Hence, we cannot reproduce the results of Rebbeck et al. ${ }^{18}$ Several individuals were reported with more than 28 repeats from the study of Kadouri and coworkers, ${ }^{22}$ and even among those individuals, no effect of the longer $A R$ CAG repeats on breast cancer phenotype could be shown. Taken together with the result for the possible effects of the short allele $A R$ CAG repeats, it seems obvious that the issue is complex and needs to be addressed in much larger study.

\section{References}

1 Tonin P, Weber B, Offit K et al: Frequency of recurrent BRCA1 and BRCA2 mutations in Ashkenazi Jewish breast cancer families. Nat Med 1996; 2: 1179-1183.

2 Abeliovich D, Kaduri L, Lerer I et al: The founder mutation 185delAG and 5382insC in BRCA1 and 6174delT in BRCA2 appear in $60 \%$ of ovarian cancer and $30 \%$ of early-onset breast cancer patients among Ashkenazi women. Am J Hum Genet 1997; 60: $505-514$.

3 Couch FJ, DeShano ML, Blackwood MA et al: BRCA1 mutations in women attending clinics that evaluate the risk of breast cancer. $N$ Engl J Med 1997; 336: 1409-1415.

4 Struewing JP, Hartge P, Wacholder J et al: The risk of cancer associated with specific mutations in BRCA1 and BRCA2 among Ashkenazi Jews. N Engl J Med 1997; 336: 1401-1408.

5 Risch HA, McLaughlin JR, Cole DE et al: Prevalence and penetrance of gernline BRCA1 and BRCA2 mutations in population series of 649 women with ovarian cancer. An J Hum Genet 2001; 68: $700-710$.

6 Struewing JP, Abeliovich D, Peretz T et al: The carrier frequency of the BRCA1 185delAG mutation is approximately 1 percent in Ashkenazi Jewish individuals. Nat Genet 1995; 11: 198-200.

7 Oddoux C, Struewing JP, Clayton CM et al: The carrier frequency of the BRCA2 6174delT mutation among Ashkenazi Jewish individuals is approximately 1\%. Nat Genet 1996; 14: 188-190.

8 Roa BB, Boyd AA, Volcik K, Richards CS: Ashkenazi Jewish population frequencies for common mutations in BRCA1 and BRCA2. Nat Genet 1996; 14: 185-187.
9 Warner E, Foulks W, Goodwin P et al: Prevalence and penetrance of BRCA1 and BRCA2 gene mutations in unselected Ashkenazi Jewish women with breast cancer. J Natl Cancer Inst 1999; 91: $1241-1247$.

10 Modan B, Hartge P, Hirsch-Yechezkel G et al and the National Israel Ovarian Cancer Study Group: Parity, oral contraceptives, and the risk of ovarian cancer among carriers and non carriers of a BRCA1 or BRCA2 mutation. N Engl J Med 2001; 345: $235-$ 240.

11 Fan S, Wang J, Yuan R et al: BRCA1 inhibition of estrogen receptor signaling in transfected cells. Science 1999; 284: 1354-1356.

12 Irvine RU, Yu MC, Ross RK, Coetzee GA: The CAG and GGC microsatellites of the androgen receptor gene are in linkage disequilibirium in men with prostate cancer. Cancer Res 1995; 55: $1937-1940$.

13 Giovannucci E, Stampfer MJ, Krithivas K et al: The CAG repeat within the androgen receptor gene and its relationship to prostate cancer. Proc Acad Sci USA 1997; 94: 3320-3323.

14 Hardy DO, Scher HI, Bogenreider T et al: Androgen receptor CAG repeat lengths in prostate cancer: correlation with age of onset. $J$ Clin Endocrinol Metab 1996; 81: 4400-4405.

15 Spurdle AB, Dite GS, Chen X et al: Androgen receptor exon 1 CAG repeat length and breast cancer in women before age forty years. $J$ Natl Cancer Inst 1999; 91: 961-966.

16 Dunning AM, McBride S, Gregory J et al: No association between androgen and vitamin D receptor gene polymorphisms and risk of breast cancer. Carcinogenesis 1999; 20: 2131-2135.

17 Giguere Y, Dewailly E, Brisson J et al: Short polyglutamine tracts in the androgen receptor are protective against breast cancer in the general population. Cancer Res 2001; 61: 5869-5874.

18 Rebbeck TR, Kantoff PW, Krithivas K et al: Modification of BRCA1associated breast cancer risk by the polymorphic androgen-receptor CAG repeat. Am J Hum Genet 1999; 64: 1371-1377.

19 Rohlfs EM, Learning WG, Friedman KJ, Couch FJ, Weber BL, Silverman LM: Direct detection of mutations in the breast and ovarian cancer susceptibility gene BRCA1 by PCR-mediated sitedirected mutagenesis. Clin Chem 1997; 43: 24-29.

20 Shiri-Sverdlov R, Gershoni-Baruch R, Ichezkel-Hirsch G et al: The Tyr978X BRCA1 mutation in non-Ashkenazi Jews: occurrence in high-risk families, general population and unselected ovarian cancer patients. Community Genet 2001; 4: 50-55.

21 Szabo CI, van Eijk R, Schreiber M, Kadouri L, Csokay B, Kote-Jarai Z: Andrgen Receptor cag repeat length and BRCA1 assocoated breast cancer risk: size does matter. Presented in the International Collaborative Group on Familial Breast/Ovarian Cancer, Venice, pp 2001, (abstract \# 6).

22 Kadouri L, Easton DF, Edwards S et al: CAG and GGC repeat polymorphisms in the androgen receptor gene and breast cancer susceptibility in BRCA1/2 carriers and non-carriers. Br J Cancer 2001; 85: 36-40.

23 Levine DA, Boyd J: The androgen receptor and genetic susceptibility to ovarian cancer: results from a case series. Cancer Res 2001; 61: 908-911.

24 Chamberlain NL, Driver ED, Meisfeld RL: The length and location of CAG trinucleotide repeats in the androgen receptor N-terminal domain affect transactivation function. Nucleic Acid Res 1994; 22: $3181-3186$.

25 Birrel SN, Bentel JM, Hickey TE et al: Androgen induce divergent proliferative responses in human breast cancer cell lines. J Steroid Biochem Mol Biol 1995; 52: 459-467.

$26 \mathrm{Yu} \mathrm{H}$, Bharaj B, Vassilikos EJ, Giani M, Diamandis EP: Shorter CAG repeat length in the androgen receptor gene is associated with more aggressive forms of breast cancer. Breast Cancer Res Treat 2000; 59: 153-161. 